\title{
A combinatorial proof of a Weyl type formula for hook Schur polynomials
}

\author{
Jae-Hoon Kwon
}

Received: 1 February 2007 / Accepted: 14 November 2007 / Published online: 27 November 2007

(C) Springer Science+Business Media, LLC 2007

\begin{abstract}
In this paper, we present a simple combinatorial proof of a Weyl type formula for hook Schur polynomials, which was obtained previously by other people using a Kostant type cohomology formula for $\mathfrak{g l}_{m \mid n}$. In general, we can obtain in a combinatorial way a Weyl type character formula for various irreducible highest weight representations of a Lie superalgebra, which together with a general linear algebra forms a Howe dual pair.
\end{abstract}

Keywords Hook Schur polynomial · Lie superalgebra $\cdot$ Character formula

\section{Introduction}

The notion of hook Schur polynomial appears naturally as characters of complex irreducible tensor representations of the general linear superalgebra $\mathfrak{g l}_{m \mid n}[3,31]$. Recently, in [10] Cheng and Zhang proved a Kostant type cohomology formula for $\mathfrak{g l}_{m \mid n}$ associated to its irreducible tensor representations to compute the corresponding generalized Kazhdan-Lusztig polynomials (cf. [30]), which also implies a Weyl type character formula for irreducible tensor representations by Euler-Poincaré principle (cf. [18]). This Weyl type character formula, which is given as an alternating sum of characters of Kac modules, is closely related to a general approach to the study of the complex irreducible finite dimensional representations of $\mathfrak{g l}_{m \mid n}$ (cf. [5, 22, 30]).

In this paper, we introduce a new combinatorial proof of the Weyl type formula for hook Schur polynomials obtained in [10]. Our proof, which was originally motivated by [11], is simple and natural in the sense that we use only the classical Weyl formula

This research was supported by 2007 research fund of University of Seoul.

J.-H. Kwon $(\varangle)$

Department of Mathematics, University of Seoul, 90 Cheonnong-dong, Dongdaemun-gu, Seoul 130-743, South Korea

e-mail: jhkwon@uos.ac.kr 
and the Cauchy identity for Schur polynomials. In fact, we prove a Weyl type formula for a more general class of functions which arise naturally as characters of quasifinite irreducible representations, not necessarily finite dimensional, of various Lie (super)algebras $[8,15,17,23]$, and were also introduced in a combinatorial way in [27].

A set $S$ is called $\mathbb{Z}_{2}$-graded if there exists a function $|\cdot|: S \rightarrow \mathbb{Z}_{2}$. For $s \in S$, we call $|s|$ the degree of $s$. We put $S_{\varepsilon}=\{s \in S|| s \mid=\varepsilon\}$ for $\varepsilon \in \mathbb{Z}_{2}$. Suppose that $\mathcal{A}$ and $\mathcal{B}$ are $\mathbb{Z}_{2}$-graded sets at most countable, and $\lambda$ is a generalized partition of length $d$. Let

$$
S_{\lambda}^{\mathcal{A} / \mathcal{B}}=\sum_{\mu, v} S_{\mu}\left(\mathbf{x}_{\mathcal{A}}\right) S_{\nu}\left(\mathbf{x}_{\mathcal{B}}^{-1}\right),
$$

where $S_{\mu}\left(\mathbf{x}_{\mathcal{A}}\right)$ and $S_{v}\left(\mathbf{x}_{\mathcal{B}}^{-1}\right)$ are hook Schur functions (or super symmetric functions) in the variables $\mathbf{x}_{\mathcal{A}}=\left\{x_{a} \mid a \in \mathcal{A}\right\}$ and $\mathbf{x}_{\mathcal{B}}^{-1}=\left\{x_{b}^{-1} \mid b \in \mathcal{B}\right\}$ corresponding to skew shapes $\mu=\left(\lambda+\left(p^{d}\right)\right) / \eta$ and $v=\left(p^{d}\right) / \eta$ for some $p \geq 0$ and $\eta$ (see Definition 2.1). Then the main result (Theorem 3.10) is

$$
S_{\lambda}^{\mathcal{A} / \mathcal{B}}=\frac{\sum_{w \in \mathcal{W}}(-1)^{\ell(w)} S_{\lambda^{w,+}}\left(\mathbf{x}_{\mathcal{A}}\right) S_{\lambda^{w,-}}\left(\mathbf{x}_{\mathcal{B}}^{-1}\right)}{\Delta_{\mathcal{A} / \mathcal{B}}},
$$

where $\Delta_{\mathcal{A} / \mathcal{B}}=\prod_{|a|=|b|}\left(1-x_{a} x_{b}^{-1}\right) \prod_{|a| \neq|b|}\left(1+x_{a} x_{b}^{-1}\right)^{-1}, \mathcal{W}$ is a set of right coset representatives of an affine Weyl group of type $A_{\infty}$ with respect to a maximal parabolic subgroup, and $\lambda^{w, \pm}$ are defined under an action of $w \in \mathcal{W}$ on $\lambda$. We also give alternative proofs of a Cauchy identity of $S_{\lambda}^{\mathcal{A} / \mathcal{B}}$ paired with rational Schur polynomials and a Jacobi-Trudi identity for $S_{\lambda}^{\mathcal{A} / \mathcal{B}}$ (cf. [27]). Some of the arguments might be stated or understood more easily in the context of representation theory, but we give self-contained combinatorial proofs which do not depend on it.

Now a Weyl type character formula for hook Schur polynomials or irreducible tensor representations of $\mathfrak{g l}_{m \mid n}$ (Theorem 4.3) follows as a byproduct, up to a multiplication of a monomial, when $\mathcal{A}$ and $\mathcal{B}$ are finite sets (say $|\mathcal{A}|=n$ and $|\mathcal{B}|=m$ ) of even and odd degree, respectively. This recovers in a purely combinatorial way the character formula given in [10].

In general, we can obtain Weyl type character formulas for other irreducible highest weight representations of a Lie (super)algebra, whenever it forms a Howe dual pair with a general linear algebra (cf. [7, 8, 17, 19, 23, 28]), since the characters of the associated representations are given by $S_{\lambda}^{\mathcal{A} / \mathcal{B}}$ under suitable choices of $\mathcal{A}$ and $\mathcal{B}$ [27]. We discuss in detail one more example in representation theory when both $\mathcal{A}$ and $\mathcal{B}$ are finite sets of even degree (say $|\mathcal{A}|=n$ and $|\mathcal{B}|=m$ ). We deduce from Howe duality $[19,24]$ that the corresponding $S_{\lambda}^{\mathcal{A} / \mathcal{B}}$, up to a multiplication of a monomial, is a character of an infinite dimensional representation of $\mathfrak{g l}_{m+n}$, which is of particular importance in the study of unitary highest weight representations of the Lie group $U(m, n)$ (cf. [15]). In this case, we obtain a Weyl type character formula given as an alternating sum of characters of generalized Verma modules (Theorem 4.10), which recovers the Enright's character formula [12,14] with a different parametrization of highest weights for generalized Verma modules, and also an analogue of the JacobiTrudi formula for these infinite dimensional representations. 
Finally, we would like to mention that the similarity of character formulas for $\mathfrak{g l}_{m \mid n}$ in [10] and $\mathfrak{g l}_{m+n}$ in [14] was already observed, and recently a more direct connection between the Grothendieck groups of module categories of $\mathfrak{g l}_{m \mid n}$ and $\mathfrak{g l}_{m+n}$ has been established in [9], where the Weyl type character formula for irreducible tensor representations of $\mathfrak{g l}_{m \mid n}$ also appears as a corollary.

The paper is organized as follows. In Section 2, we recall some basic terminologies. In Section 3, we derive a Weyl type formula, a Cauchy type identity, and a Jacobi-Trudi formula for $S_{\lambda}^{\mathcal{A} / \mathcal{B}}$. We also discuss a factorization property for $S_{\lambda}^{\mathcal{A} / \mathcal{B}}$ when $\mathcal{A}$ is a finite set of even degree, which provides another proof of the factorization of hook Schur polynomials. In Section 4, we discuss applications to irreducible tensor representations of $\mathfrak{g l}_{m \mid n}$, and infinite dimensional representations of $\mathfrak{g l}_{m+n}$.

\section{Symmetric functions}

Let us recall some terminologies (cf. [29]). A partition is a non-increasing sequence of non-negative integers $\lambda=\left(\lambda_{k}\right)_{k \geq 1}$ such that $\sum_{k \geq 1} \lambda_{k}<\infty$. The number of non-zero parts in $\lambda$ is called the length of $\lambda$ denoted by $\ell(\lambda)$. We also write $\lambda=\left(1^{m_{1}}, 2^{m_{2}}, \ldots\right)$, where $m_{i}$ is the number of parts equal to $i$. We denote by $\mathcal{P}$ the set of all partitions. A partition $\lambda=\left(\lambda_{k}\right)_{k \geq 1}$ is identified with a Young diagram which is a collection of nodes (or boxes) in left-justified rows with $\lambda_{k}$ nodes in the $k$ th row. We denote by $\lambda^{\prime}$ the conjugate of $\lambda$. For $\lambda, \mu \in \mathcal{P}$, let $\lambda+\mu=\left(\lambda_{k}+\mu_{k}\right)_{k \geq 1}$, and if $\lambda \supset \mu$ (that is, $\lambda_{k} \geq \mu_{k}$ for all $k$ ), let $\lambda / \mu$ be the skew Young diagram obtained from $\lambda$ by removing $\mu$.

For a set $K$ which is at most countable, let $\Lambda_{K}$ be the ring of symmetric functions in the variables $\mathbf{x}_{K}=\left\{x_{k} \mid k \in K\right\}$, and $s_{\lambda}\left(\mathbf{x}_{K}\right)$ the Schur function corresponding to $\lambda \in \mathcal{P}$. When $K$ is infinite, let $\omega_{K}$ be the involution on $\Lambda_{K}$, which sends $s_{\lambda}\left(\mathbf{x}_{K}\right)$ to $s_{\lambda^{\prime}}\left(\mathbf{x}_{K}\right)$.

Throughout the paper, we assume that $\mathcal{A}$ and $\mathcal{B}$ are $\mathbb{Z}_{2}$-graded sets, which are at most countable. We put $\mathbb{Z}=\{0, \pm 1, \pm 2, \ldots\}, \mathbb{Z}_{>0}=\{1,2, \ldots\}, \mathbb{Z}_{<0}=$ $\{-1,-2, \ldots\},[n]=\{1, \ldots, n\}$, and $[-n]=\{-1, \ldots,-n\}(n \geq 1)$, where all the elements are assumed to be of degree 0 (or even). Also, we define $\mathcal{A}^{\prime}=\left\{a^{\prime} \mid a \in \mathcal{A}\right\}$ to be the set with the opposite $\mathbb{Z}_{2}$-grading, that is, $\left|a^{\prime}\right| \equiv|a|+1(\bmod 2)$ for $a \in \mathcal{A}$.

Let $\mathbf{x}_{\mathcal{A}}=\left\{x_{a} \mid a \in \mathcal{A}\right\}$ be the set of variables indexed by $\mathcal{A}$ and $\mathbf{x}_{\mathcal{A}}^{-1}=\left\{x_{a}^{-1} \mid\right.$ $a \in \mathcal{A}\}$. For a skew Young diagram $\lambda / \mu$, a hook Schur function corresponding to $\lambda / \mu$ is defined to be

$$
S_{\lambda / \mu}\left(\mathbf{x}_{\mathcal{A}}\right)=\sum_{\substack{\nu \in \mathcal{P} \\ \mu \subset \mathcal{V} \subset \lambda}} s_{\mathcal{V} / \mu}\left(\mathbf{x}_{\mathcal{A}_{0}}\right) s_{\lambda^{\prime} / \nu^{\prime}}\left(\mathbf{x}_{\mathcal{A}_{1}}\right)
$$

(cf. [3, 29]). For simplicity, we shall often write $S_{\lambda / \mu}^{\mathcal{A}}=S_{\lambda / \mu}\left(\mathbf{x}_{\mathcal{A}}\right)$. When $\mathcal{A}$ is finite, $S_{\lambda}^{\mathcal{A}}$ is a hook Schur polynomial introduced by Berele and Regev [3]. Following our notation, we may write $S_{\lambda}^{\mathcal{A}}=\sum_{\mu \in \mathcal{P}} S_{\mu}^{\mathcal{A}_{0}} S_{\lambda / \mu}^{\mathcal{A}_{1}}$ for $\lambda \in \mathcal{P}$, and hence $S_{\lambda}^{\mathcal{A}}=s_{\lambda}\left(\mathbf{x}_{\mathcal{A}}\right)$ if $\mathcal{A}=\mathcal{A}_{0}$, and $S_{\lambda}^{\mathcal{A}}=s_{\lambda^{\prime}}\left(\mathbf{x}_{\mathcal{A}}\right)$ if $\mathcal{A}=\mathcal{A}_{1}$. 
For a positive integer $d$, let $\mathbb{Z}_{+}^{d}=\left\{\lambda=\left(\lambda_{1}, \cdots, \lambda_{d}\right) \in \mathbb{Z}^{d} \mid \lambda_{1} \geq \cdots \geq \lambda_{d}\right\}$ be the set of generalized partitions of length $d$. For $\lambda \in \mathbb{Z}_{+}^{d}$, put

$$
\begin{aligned}
& \lambda^{+}=\left(\max \left(\lambda_{1}, 0\right), \ldots, \max \left(\lambda_{d}, 0\right)\right) \in \mathcal{P}, \\
& \lambda^{-}=\left(\max \left(-\lambda_{d}, 0\right), \ldots, \max \left(-\lambda_{1}, 0\right)\right) \in \mathcal{P}, \\
& \lambda^{*}=\left(-\lambda_{d}, \ldots,-\lambda_{1}\right) \in \mathbb{Z}_{+}^{d} .
\end{aligned}
$$

The addition on $\mathbb{Z}_{+}^{d}$ is defined in a usual way, and then $\lambda=\lambda^{+}+\left(\lambda^{-}\right)^{*}$.

Definition 2.1 ([27]) For $\lambda \in \mathbb{Z}_{+}^{d}$, we define

$$
S_{\lambda}\left(\mathbf{x}_{\mathcal{A}} ; \mathbf{x}_{\mathcal{B}}\right)=\sum_{\mu, v} S_{\mu}\left(\mathbf{x}_{\mathcal{A}}\right) S_{\nu}\left(\mathbf{x}_{\mathcal{B}}^{-1}\right)
$$

where $\mu$ and $\nu$ are skew Young diagrams of the form

$$
\mu=\left(\lambda+\left(p^{d}\right)\right) / \eta, \quad v=\left(p^{d}\right) / \eta
$$

for some non-negative integer $p$ and partition $\eta$ such that $\lambda+\left(p^{d}\right) \in \mathcal{P}, \eta \subset \lambda+\left(p^{d}\right)$, and $\eta \subset\left(p^{d}\right)$. Let us write $S_{\lambda}^{\mathcal{A} / \mathcal{B}}=S_{\lambda}\left(\mathbf{x}_{\mathcal{A}} ; \mathbf{x}_{\mathcal{B}}\right)$ for simplicity.

Remark 2.2 (1) If $\mathcal{B}$ is empty, then $S_{\lambda}^{\mathcal{A} / \mathcal{B}}$ is non-zero only if $\lambda$ is an ordinary partition, and in this case, we have $S_{\lambda}^{\mathcal{A} / \mathcal{B}}=S_{\lambda}^{\mathcal{A}}$.

(2) By definition, $S_{\lambda}^{\mathcal{A} / \mathcal{B}}$ can be regarded as the character of certain bitableaux. A combinatorics of these bitableaux, including analogues of the Schensted insertion, the Littlewood-Richardson rule, and the Robinson-Schensted-Knuth correspondence, are developed in [27].

For $\lambda \in \mathbb{Z}_{+}^{d}$, let $s_{\lambda}\left(\mathbf{x}_{[d]}\right)$ be the rational (or Laurent) Schur polynomial corresponding to $\lambda$, that is, $s_{\lambda}\left(\mathbf{x}_{[d]}\right)=\left(x_{1} \cdots x_{d}\right)^{-p} s_{\lambda+\left(p^{d}\right)}\left(\mathbf{x}_{[d]}\right)$ for $p \geq 0$ such that $\lambda+\left(p^{d}\right) \in \mathcal{P}$. For $\mu, v \in \mathbb{Z}_{+}^{d}$, we have

$$
s_{\mu}\left(\mathbf{x}_{[d]}\right) s_{\nu}\left(\mathbf{x}_{[d]}\right)=\sum_{\lambda \in \mathbb{Z}_{+}^{d}} c_{\mu \nu}^{\lambda} s_{\lambda}\left(\mathbf{x}_{[d]}\right),
$$

where $c_{\mu \nu}^{\lambda}$ is a Littlewood-Richardson coefficient. Note that $c_{\mu \nu}^{\lambda}=c_{\mu+\left(p^{d}\right) v+\left(q^{d}\right)}^{\left.\lambda+(p+q)^{d}\right)}$ for all $p, q \geq 0$. Then we have another expression of $S_{\lambda}^{\mathcal{A} / \mathcal{B}}$ as a linear combination of the products $S_{\mu}\left(\mathbf{x}_{\mathcal{A}}\right) S_{\nu}\left(\mathbf{x}_{\mathcal{B}}^{-1}\right)$ for $\mu, v \in \mathcal{P}$.

Proposition 2.3 ([27]) For $\lambda \in \mathbb{Z}_{+}^{d}$, we have

$$
S_{\lambda}\left(\mathbf{x}_{\mathcal{A}} ; \mathbf{x}_{\mathcal{B}}\right)=\sum_{\substack{\mu, v \in \mathcal{P} \\ \ell(\mu), \ell(v) \leq d}} c_{\mu v^{*}}^{\lambda} S_{\mu}\left(\mathbf{x}_{\mathcal{A}}\right) S_{\nu}\left(\mathbf{x}_{\mathcal{B}}^{-1}\right) .
$$




\section{Weyl type formula}

\subsection{Main result}

Put $\mathbb{Z}^{\times}=\mathbb{Z} \backslash\{0\}$. Let $P=\bigoplus_{i \in \mathbb{Z}^{\times}} \mathbb{Z} \epsilon_{i}$ be the free Abelian group generated by $\left\{\epsilon_{i} \mid i \in\right.$ $\left.\mathbb{Z}^{\times}\right\}$. For $i \in \mathbb{Z}$, let $r_{i}$ be the transposition on $\mathbb{Z}^{\times}$(hence on $\left\{\epsilon_{i} \mid i \in \mathbb{Z}^{\times}\right\}$) given by

$$
r_{i}= \begin{cases}(i i+1), & \text { if } i>0, \\ (i-1 i), & \text { if } i<0, \\ (-11), & \text { if } i=0 .\end{cases}
$$

Let $W$ be the Coxeter group generated by $\left\{r_{i} \mid i \in \mathbb{Z}\right\}$, and $\ell(w)$ denote the length of $w \in W$. For each subset $I \subset \mathbb{Z}$, let $W_{I}$ be the subgroup of $W$ generated by $\left\{r_{i} \mid i \in I\right\}$. Let

$$
\mathcal{W}=\left\{w \in W \mid \ell\left(r_{i} w\right)>\ell(w) \text { for } i \in \mathbb{Z}^{\times}\right\},
$$

be the set of right coset representatives with respect to a maximal parabolic subgroup $W_{\mathbb{Z}^{\times}}$(cf. [4]).

For $\lambda \in \mathbb{Z}_{+}^{d}$ and $w \in \mathcal{W}$, choose sufficiently large $p, q>0$ such that

(1) $-p \leq \lambda_{d} \leq \lambda_{1} \leq q$,

(2) $w \in W_{I(p, q)}$, where $I(p, q)=\{k \in \mathbb{Z} \mid-p+1 \leq k \leq q-1\}$.

Put $\mu=\left(\lambda+\left(p^{d}\right)\right)^{\prime}=\left(\mu_{1}, \ldots, \mu_{n}\right)$, where $n=p+q$. We may identify $\mu$ with

$$
\mu=\mu_{1} \epsilon_{-p}+\cdots+\mu_{p} \epsilon_{-1}+\mu_{p+1} \epsilon_{1}+\cdots+\mu_{p+q} \epsilon_{q} \in P .
$$

Then, we define

$$
w \circ \lambda=w\left(\mu+\rho_{p, q}\right)-\rho_{p, q}-d \mathbf{1}_{p},
$$

where $\rho_{p, q}=\sum_{i \in[-p]}(q-i-1) \epsilon_{i}+\sum_{j \in[q]}(q-j) \epsilon_{j}$, and $\mathbf{1}_{p}=\sum_{i \in[-p]} \epsilon_{i}$.

Lemma 3.1 Under the above hypothesis, there exist unique $\sigma, \tau \in \mathcal{P}$ with $\ell(\sigma) \leq p$ and $\ell(\tau) \leq q$ such that

$$
w \circ \lambda=-\sum_{i \in \mathbb{Z}_{<0}} \sigma_{-i} \epsilon_{i}+\sum_{j \in \mathbb{Z}_{>0}} \tau_{j} \epsilon_{j} .
$$

Proof First, let $w\left(\rho_{p, q}\right)-\rho_{p, q}=\sum_{i \in \mathbb{Z}^{\times}} a_{i} \epsilon_{i} \in P$, where $a_{i}=0$ for $i \notin[-p] \cup[q]$. It is not difficult to see that $w\left(\rho_{p^{\prime}, q^{\prime}}\right)-\rho_{p^{\prime}, q^{\prime}}=w\left(\rho_{p, q}\right)-\rho_{p, q}$ for all $p^{\prime}>p$ and $q^{\prime}>q$. Since $w$ is a right coset representative of $W_{\mathbb{Z}^{\times}}$in $W$, we also have $a_{i} \geq a_{i+1}$ and $a_{-i-1} \geq a_{-i}$ for all $i>0$ (cf. [4]). This implies that

$$
\begin{aligned}
& 0 \geq a_{-p} \geq a_{-p+1} \geq \ldots \geq a_{-1}, \\
& a_{1} \geq a_{2} \geq \ldots \geq a_{q} \geq 0 .
\end{aligned}
$$

Next, if we put $w(\mu)=\sum_{i \in[-p] \cup[q]} b_{i} \epsilon_{i}$, then we have

$$
d \geq b_{-p} \geq \cdots \geq b_{-1} \geq 0, \quad b_{1} \geq \cdots \geq b_{q} \geq 0 .
$$


Note that $w(\mu)-d \mathbf{1}_{p}$ does not depend on the choice of $p, q$. Hence it follows that $w \circ \lambda=\sum_{i \in[-p] \cup[q]} c_{i} \epsilon_{i}$, where

$$
\begin{aligned}
& 0 \geq c_{-p} \geq c_{-p+1} \geq \ldots \geq c_{-1}, \\
& c_{1} \geq c_{2} \geq \ldots \geq c_{q} \geq 0 .
\end{aligned}
$$

This completes the proof.

Definition 3.2 For $\lambda \in \mathbb{Z}_{+}^{d}$ and $w \in \mathcal{W}$, we define

$$
\lambda^{w,-}=\sigma^{\prime}, \quad \lambda^{w,+}=\tau^{\prime},
$$

where $\sigma, \tau \in \mathcal{P}$ are given in Lemma 3.1.

Remark 3.3 (1) Given $w \in \mathcal{W}$, suppose that $w \in W_{I(p, q)}$ for some $p, q>0$. If $w\left(\rho_{p, q}\right)-\rho_{p, q}=\sum_{i \in \mathbb{Z}^{\times}} \mu_{i} \epsilon_{i} \in P$, then we can check that the partition $\left(-\mu_{-k}\right)_{k \geq 1}$ is the conjugate of $\left(\mu_{k}\right)_{k \geq 1}$ (see 2.4 in [4]). Moreover, the map sending $w$ to $\left(\mu_{k}\right)_{k \geq 1}$ is a one-to-one correspondence between $\mathcal{W}$, the set of the minimal length right coset representatives and $\mathcal{P}$, where $\ell(w)=|\mu|=\sum_{k \geq 1} \mu_{k}$.

(2) One can also check that for $\lambda \in \mathbb{Z}_{+}^{d},\left(\lambda^{w,-}, \lambda^{w,+}\right)=\left(\lambda^{w^{\prime},-}, \lambda^{w^{\prime},+}\right)$ if and only if $w=w^{\prime} \in \mathcal{W}$.

Next, consider the Schur polynomials in $n$ variables. Fix $p, q>0$ such that $p+$ $q=n$. Instead of $[n]$, let us use $[-p, q]=[-p] \cup[q]$ as an index set for the variables. For a partition $\mu$ with $\ell(\mu) \leq n$, we may identify $\mu$ with

$$
\mu_{1} \epsilon_{-p}+\cdots+\mu_{p} \epsilon_{-1}+\mu_{p+1} \epsilon_{1}+\cdots+\mu_{p+q} \epsilon_{q} \in P .
$$

Then $W_{I(p, q)}$, which is isomorphic to the symmetric group on $n$ letters, naturally acts on $\mu$. Given $\alpha=\sum_{i \in[-p, q]} c_{i} \epsilon_{i} \in P$, put $\mathbf{x}_{[-p, q]}^{\alpha}=\prod_{i \in[-p, q]} x_{i}^{c_{i}}$. Recall that the Weyl formula for the Schur polynomial corresponding to $\mu$ is given by

$$
s_{\mu}\left(\mathbf{x}_{[-p, q]}\right)=\frac{\sum_{w \in W_{I(p, q)}}(-1)^{\ell(w)} \mathbf{x}_{[-p, q]}^{w\left(\mu+\rho_{p, q}\right)-\rho_{p, q}}}{\prod_{i, j \in[-p, q], i<j}\left(1-x_{i}^{-1} x_{j}\right)} .
$$

Then we have a parabolic analogue as follows.

Lemma 3.4 Suppose that $\mu \subset\left(d^{n}\right)$ for some $d>0$, and $p+q=n$. Following the above notations, we have

$$
\mathbf{x}_{[-p, q]}^{-d \mathbf{1}_{p}} s_{\mu}\left(\mathbf{x}_{[-p, q]}\right)=\frac{\sum_{w \in \mathcal{W} \cap W_{I(p, q)}}(-1)^{\ell(w)} s_{(\lambda w,+)^{\prime}}\left(\mathbf{x}_{[q]}\right) s_{(\lambda w,-)^{\prime}}\left(\mathbf{x}_{[-p]}^{-1}\right)}{\prod_{i \in[-p]} \prod_{j \in[q]}\left(1-x_{i}^{-1} x_{j}\right)},
$$

where $\lambda=\mu^{\prime}-\left(p^{d}\right) \in \mathbb{Z}_{+}^{d}$ and $\lambda^{w, \pm}$ are defined in (3.7). 
Proof By Lemma 3.1, we have for $w \in \mathcal{W} \cap W_{I(p, q)}$,

$$
w\left(\mu+\rho_{p, q}\right)-\rho_{p, q}-d \mathbf{1}_{p}=-\sum_{i \in[-p]} \sigma_{-i} \epsilon_{i}+\sum_{j \in[q]} \tau_{j} \epsilon_{j},
$$

for some $\sigma, \tau \in \mathcal{P}$ with $\ell(\sigma) \leq p$ and $\ell(\tau) \leq q$. Now, for $w^{\prime} \in W_{I(p, q) \backslash\{0\}}$, we have

$$
\begin{aligned}
w^{\prime} & w\left(\mu+\rho_{p, q}\right)-\rho_{p, q}-d \mathbf{1}_{p} \\
& =w^{\prime}\left(w\left(\mu+\rho_{p, q}\right)-\rho_{p, q}-d \mathbf{1}_{p}+\rho_{p, q}\right)-\rho_{p, q} \\
& =w^{\prime}\left(-\sum_{i \in[-p]} \sigma_{-i} \epsilon_{i}+\sum_{j \in[q]} \tau_{j} \epsilon_{j}+q \mathbf{1}_{p}+\rho_{p}^{-}+\rho_{q}^{+}\right)-q \mathbf{1}_{p}-\rho_{p}^{-}-\rho_{q}^{+} \\
& =w^{\prime}\left(-\sum_{i \in[-p]} \sigma_{-i} \epsilon_{i}+\sum_{j \in[q]} \tau_{j} \epsilon_{j}+\rho_{p}^{-}+\rho_{q}^{+}\right)-\rho_{p}^{-}-\rho_{q}^{+},
\end{aligned}
$$

where $\rho_{p}^{-}=\sum_{i \in[-p]}(-i-1) \epsilon_{i}, \rho_{q}^{+}=\sum_{j \in[q]}(q-j) \epsilon_{j}$ and $\rho_{p, q}=q \mathbf{1}_{p}+\rho_{p}^{-}+\rho_{q}^{+}$. Since $s_{v}\left(\mathbf{x}_{[-p]}\right)=s_{v^{*}}\left(\mathbf{x}_{[-p]}^{-1}\right)$ for $v \in \mathbb{Z}_{+}^{p}$, we obtain the result from (3.8).

In terms of $S_{\lambda}^{\mathcal{A} / \mathcal{B}}$, Lemma 3.4 can be written as follows.

Lemma 3.5 For $\lambda \in \mathbb{Z}_{+}^{d}$, choose $p, q>0$ such that $-p \leq \lambda_{d} \leq \lambda_{1} \leq q$. Then we have

$$
S_{\lambda}^{[q]^{\prime} /[-p]^{\prime}}=\frac{\sum_{w \in \mathcal{W} \cap W_{I(p, q)}}(-1)^{\ell(w)} S_{\lambda^{w,+}}\left(\mathbf{x}_{[q]^{\prime}}\right) S_{\lambda w,-}\left(\mathbf{x}_{[-p]^{\prime}}^{-1}\right)}{\prod_{i \in[-p]^{\prime}} \prod_{j \in[q]^{\prime}}\left(1-x_{i}^{-1} x_{j}\right)} .
$$

Proof Put $\mu=\left(\lambda+\left(p^{d}\right)\right)^{\prime}$. Note that $S_{\eta}\left(\mathbf{x}_{[q]^{\prime}}\right)=s_{\eta^{\prime}}\left(\mathbf{x}_{[q]^{\prime}}\right)$ and $S_{\eta}\left(\mathbf{x}_{[-p]^{]^{\prime}}}^{-1}\right)=$ $s_{\eta^{\prime}}\left(\mathbf{x}_{[-p]^{\prime}}^{-1}\right)$ for $\eta \in \mathcal{P}$. Then, we have

$$
\begin{aligned}
\mathbf{x}_{[-p, q]^{\prime}}^{-d \mathbf{1}_{p}} s_{\mu}\left(\mathbf{x}_{[-p, q]^{\prime}}\right) & =\sum_{\nu} \mathbf{x}_{[-p, q]^{\prime}}^{-d \mathbf{1}_{p}} s_{\nu}\left(\mathbf{x}_{[-p]^{\prime}}\right) s_{\mu / v}\left(\mathbf{x}_{[q]^{\prime}}\right) \\
& =\sum_{\nu} s_{\nu-\left(d^{p}\right)}\left(\mathbf{x}_{[-p]^{\prime}}\right) s_{\mu / v}\left(\mathbf{x}_{[q]^{\prime}}\right) \\
& =\sum_{\nu} s_{\left(d^{p}\right) / v}\left(\mathbf{x}_{[-p]^{\prime}}^{-1}\right) s_{\mu / v}\left(\mathbf{x}_{[q]^{\prime}}\right) \\
& =\sum_{\eta} S_{\left(p^{d}\right) / \eta}\left(\mathbf{x}_{[-p]^{\prime}}^{-1}\right) S_{\left(\lambda+\left(p^{d}\right)\right) / \eta}\left(\mathbf{x}_{[q]^{\prime}}\right) \\
& =S_{\lambda}\left(\mathbf{x}_{[q]^{\prime}} ; \mathbf{x}_{[-p]^{\prime}}\right) .
\end{aligned}
$$

The result follows from Lemma 3.4 by replacing $\mathbf{x}_{[-p, q]}$ with $\mathbf{x}_{[-p, q]^{\prime}}$. 
Proposition 3.6 For $\lambda \in \mathbb{Z}_{+}^{d}$, we have

$$
S_{\lambda}^{\mathbb{Z}_{>0}^{\prime} / \mathbb{Z}_{<0}^{\prime}}=\frac{\sum_{w \in \mathcal{W}}(-1)^{\ell(w)} S_{\lambda w,+}\left(\mathbf{x}_{\mathbb{Z}_{>0}^{\prime}}\right) S_{\lambda w,-}\left(\mathbf{x}_{\mathbb{Z}_{<0}^{\prime}}^{-1}\right)}{\prod_{i, j}\left(1-x_{i}^{-1} x_{j}\right)},
$$

and a Cauchy type identity

$$
\prod_{i, j, k}\left(1+x_{i}^{-1} z_{k}^{-1}\right)\left(1+x_{j} z_{k}\right)=\sum_{\lambda \in \mathbb{Z}_{+}^{d}} S_{\lambda}^{\mathbb{Z}_{>0}^{\prime} / \mathbb{Z}_{<0}^{\prime}} s_{\lambda}\left(\mathbf{z}_{[d]}\right),
$$

where $i \in \mathbb{Z}_{<0}^{\prime}, j \in \mathbb{Z}_{>0}^{\prime}, k \in[d]$, and $\mathbf{z}_{[d]}=\left\{z_{k} \mid k \in[d]\right\}$.

Proof First, it is easy to see that for $p, q>0 S_{\lambda}^{[q+1]^{\prime} /[-p-1]^{\prime}}$ reduces to $S_{\lambda}^{[q]^{\prime} /[-p]^{\prime}}$ when we put $x_{q+1}=0$ and $x_{-p-1}^{-1}=0$. Hence, $S_{\lambda}^{[q]^{\prime} /[-p]^{\prime}}$ has the well-defined limit with respect to both $\mathbf{x}_{[q]}$ and $\mathbf{x}_{[-p]}^{-1}$ when we let $p, q \rightarrow \infty$, which is equal to

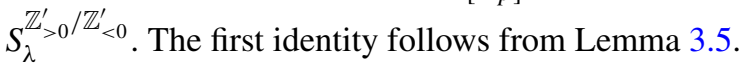

Next, consider the following dual Cauchy identity (cf. [29]).

$$
\prod_{i \in[n]} \prod_{j \in[d]}\left(1+x_{i} z_{j}\right)=\sum_{\mu \subset\left(d^{n}\right)} s_{\mu}\left(\mathbf{x}_{[n]}\right) s_{\mu^{\prime}}\left(\mathbf{z}_{[d]}\right) .
$$

Choose $p, q>0$ such that $p+q=n$. Replacing $[n]$ with $[-p, q]^{\prime}$ and multiplying $\left(x_{-p^{\prime}} \cdots x_{-1^{\prime}}\right)^{-d}\left(z_{1} \cdots z_{d}\right)^{-p}$ on both sides, we have

$$
\begin{aligned}
& \prod_{i \in[-p]^{\prime}} \prod_{j \in[q]^{]^{\prime}}} \prod_{k \in[d]}\left(1+x_{i}^{-1} z_{k}^{-1}\right)\left(1+x_{j} z_{k}\right) \\
& =\sum_{\lambda \in \mathbb{Z}_{+}^{d}} \mathbf{x}_{[-p]^{\prime}}^{-d \mathbf{1}_{p}} s_{\left(\lambda+\left(p^{d}\right)\right)^{\prime}}\left(\mathbf{x}_{[-p, q]^{\prime}}\right) s_{\lambda}\left(\mathbf{z}_{[d]}\right) \\
& =\sum_{\lambda \in \mathbb{Z}_{+}^{d}} S_{\lambda}^{[q]^{\prime} /[-p]^{\prime}} s_{\lambda}\left(\mathbf{z}_{[d]}\right) . \quad(\operatorname{see}(3.10))
\end{aligned}
$$

Hence, by letting $p, q \rightarrow \infty$, we obtain the second identity.

Proposition 3.7 For $\lambda \in \mathbb{Z}_{+}^{d}$, we have

$$
S_{\lambda}^{\mathbb{Z}_{>0} / \mathbb{Z}_{<0}}=\frac{\sum_{w \in \mathcal{W}}(-1)^{\ell(w)} S_{\lambda w,+}\left(\mathbf{x}_{\mathbb{Z}_{>0}}\right) S_{\lambda w,-}\left(\mathbf{x}_{\mathbb{Z}_{<0}^{-1}}\right)}{\prod_{i, j}\left(1-x_{i}^{-1} x_{j}\right)},
$$

and a Cauchy type identity

$$
\frac{1}{\prod_{i, j, k}\left(1-x_{i}^{-1} z_{k}^{-1}\right)\left(1-x_{j} z_{k}\right)}=\sum_{\lambda \in \mathbb{Z}_{+}^{d}} S_{\lambda}^{\mathbb{Z}_{>0} / \mathbb{Z}_{<0}} s_{\lambda}\left(\mathbf{z}_{[d]}\right),
$$

where $i \in \mathbb{Z}_{<0}, j \in \mathbb{Z}_{>0}$, and $k \in[d]$. 
Proof Applying both $\omega_{\mathbb{Z}_{<0}^{\prime}}$ and $\omega_{\mathbb{Z}_{>0}^{\prime}}$ in Proposition 3.6 and then replacing $\mathbf{x}_{\mathbb{Z}_{>0}^{\prime}}$ (resp. $\mathbf{x}_{\mathbb{Z}_{<0}^{\prime}}$ ) by $\mathbf{x}_{\mathbb{Z}_{>0}}$ (resp. $\mathbf{x}_{\mathbb{Z}_{<0}}$ ), we obtain the identities.

As a special case of Proposition 3.7, we obtain the following Cauchy type identity with a restriction on the length of partitions.

Corollary 3.8 For $d \geq 1$, we have

$$
\sum_{\substack{\lambda \in \mathcal{P} \\ \ell(\lambda) \leq d}} s_{\lambda}(\mathbf{x}) s_{\lambda}(\mathbf{y})=\frac{\sum_{w \in \mathcal{W}}(-1)^{\ell(w)} s_{\mathbf{0}_{d}^{w,+}}(\mathbf{x}) s_{\mathbf{0}_{d}^{w,-}}(\mathbf{y})}{\prod_{i, j \geq 1}\left(1-x_{i} y_{j}\right)},
$$

where $\mathbf{x}=\mathbf{x}_{\mathbb{Z}_{>0}}, \mathbf{y}=\mathbf{y}_{\mathbb{Z}_{>0}}$, and $\mathbf{0}_{d}=(0, \ldots, 0) \in \mathbb{Z}_{+}^{d}$.

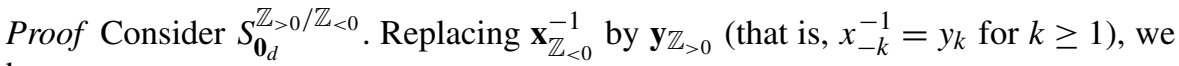
have

$$
S_{\mathbf{0}_{d}}^{\mathbb{Z}_{>0} / \mathbb{Z}_{<0}}=\sum_{\substack{\nu=\left(k^{d}\right) / \eta \\ k \geq 0, \eta \subset\left(k^{d}\right)}} s_{\nu}(\mathbf{x}) s_{\nu}(\mathbf{y})=\sum_{\ell(\lambda) \leq d} s_{\lambda}(\mathbf{x}) s_{\lambda}(\mathbf{y})
$$

since $s_{\left(k^{d}\right) / \eta}(\mathbf{x})=s_{\lambda}(\mathbf{x})$, where $\lambda=\left(k^{d}\right)+\eta^{*} \in \mathcal{P}$ (we regard $\eta$ as an element in $\mathbb{Z}_{+}^{d}$ ) by the Littlewood-Richardson rule. Combining with Proposition 3.7, we obtain the identity.

Remark 3.9 Corollary 3.8 can be stated more explicitly. Given $w \in \mathcal{W}$, let $\mu=$ $(\alpha \mid \beta)$ be the corresponding partition (see Remark 3.3) given in Frobenius notation with $\delta(\mu)$ the length of $\alpha$ or $\beta$. Then it is not difficult to see that

$$
\mathbf{0}_{d}^{w,+}=\mu+\left(d^{\delta(\mu)}\right), \quad \mathbf{0}_{d}^{w,-}=\mu^{\prime}+\left(d^{\delta(\mu)}\right) .
$$

Hence, we obtain an alternative expression

$$
\sum_{\substack{\lambda \in \mathcal{P} \\ \ell(\lambda) \leq d}} s_{\lambda}(\mathbf{x}) s_{\lambda}(\mathbf{y})=\frac{\sum_{\mu \in \mathcal{P}}(-1)^{|\mu|} s_{\mu+\left(d^{\delta(\mu)}\right)}(\mathbf{x}) s_{\mu^{\prime}+\left(d^{\delta(\mu)}\right)}(\mathbf{y})}{\prod_{i, j \geq 1}\left(1-x_{i} y_{j}\right)}
$$

Theorem 3.10 For $\lambda \in \mathbb{Z}_{+}^{d}$, we have

$$
S_{\lambda}^{\mathcal{A} / \mathcal{B}}=\frac{\sum_{w \in \mathcal{W}}(-1)^{\ell(w)} S_{\lambda^{w,+}}\left(\mathbf{x}_{\mathcal{A}}\right) S_{\lambda^{w,-}}\left(\mathbf{x}_{\mathcal{B}}^{-1}\right)}{\Delta_{\mathcal{A} / \mathcal{B}}},
$$

where

$$
\Delta_{\mathcal{A} / \mathcal{B}}=\frac{\prod_{|a|=|b|}\left(1-x_{a} x_{b}^{-1}\right)}{\prod_{|a| \neq|b|}\left(1+x_{a} x_{b}^{-1}\right)}
$$


We also have the following Cauchy type identity

$$
\prod_{k \in[d]} \frac{\prod_{a \in \mathcal{A}_{1}}\left(1+x_{a} z_{k}\right) \prod_{b \in \mathcal{B}_{1}}\left(1+x_{b}^{-1} z_{k}^{-1}\right)}{\prod_{a \in \mathcal{A}_{0}}\left(1-x_{a} z_{k}\right) \prod_{b \in \mathcal{B}_{0}}\left(1-x_{b}^{-1} z_{k}^{-1}\right)}=\sum_{\lambda \in \mathbb{Z}_{+}^{d}} S_{\lambda}^{\mathcal{A} / \mathcal{B}} s_{\lambda}\left(\mathbf{z}_{[d]}\right) .
$$

Proof For convenience, let us assume that $\mathcal{A} \subset \mathbb{Z}_{>0}$ and $\mathcal{B} \subset \mathbb{Z}_{<0}$ with arbitrary $\mathbb{Z}_{2}$ gradings. Let $\mathcal{A}^{\circ}$ (resp. $\mathcal{B}^{\circ}$ ) be the set of all positive (resp. negative) integers with a $\mathbb{Z}_{2}$-grading such that $\mathcal{A}_{i}^{\circ}$ (resp. $\mathcal{B}_{i}^{\circ}$ ) is infinite and $\mathcal{A}_{i} \subset \mathcal{A}_{i}^{\circ}$ (resp. $\mathcal{B}_{i} \subset \mathcal{B}_{i}^{\circ}$ ) for $i \in \mathbb{Z}_{2}$.

We may view $\Lambda_{\mathbb{Z}_{>0}}\left(\right.$ resp. $\Lambda_{\mathbb{Z}_{<0}}$ ) as a subring of $\Lambda_{\mathcal{A}_{0}^{\circ}} \otimes \Lambda_{\mathcal{A}_{1}^{\circ}}\left(\right.$ resp. $\Lambda_{\mathcal{B}_{0}^{\circ}} \otimes \Lambda_{\mathcal{B}_{1}^{\circ}}$ ). Applying $\omega_{\mathcal{A}_{1}^{\circ}}$ and $\omega_{\mathcal{B}_{1}^{\circ}}$ to $S_{\lambda}^{\mathbb{Z}_{>0} / \mathbb{Z}_{<0}}$ and the Cauchy type identity in Proposition 3.7 (we assume that the set of variables in $\Lambda_{\mathbb{Z}_{<0}}$ is $\mathbf{x}_{\mathbb{Z}_{<0}^{-1}}^{-}$), we obtain

$$
\begin{aligned}
& S_{\lambda}^{\mathcal{A}^{\circ} / \mathcal{B}^{\circ}}=\frac{\sum_{w \in \mathcal{W}}(-1)^{\ell(w)} S_{\lambda^{w,+}}\left(\mathbf{x}_{\mathcal{A}^{\circ}}\right) S_{\lambda^{w,-}}\left(\mathbf{x}_{\mathcal{B}^{\circ}}^{-1}\right)}{\Delta_{\mathcal{A}^{\circ} / \mathcal{B}^{\circ}}}, \\
& \prod_{k \in[d]} \frac{\prod_{a \in \mathcal{A}_{1}^{\circ}}\left(1+x_{a} z_{k}\right) \prod_{b \in \mathcal{B}_{1}^{\circ}}\left(1+x_{b}^{-1} z_{k}^{-1}\right)}{\prod_{a \in \mathcal{A}_{0}^{\circ}}\left(1-x_{a} z_{k}\right) \prod_{b \in \mathcal{B}_{0}^{\circ}}\left(1-x_{b}^{-1} z_{k}^{-1}\right)}=\sum_{\lambda \in \mathbb{Z}_{+}^{d}} S_{\lambda}^{\mathcal{A}^{\circ} / \mathcal{B}^{\circ}}{ }_{S_{\lambda}\left(\mathbf{z}_{[n]}\right) .}
\end{aligned}
$$

Finally, by letting $x_{a}=x_{b}^{-1}=0$ for $a \in \mathcal{A}^{\circ} \backslash \mathcal{A}$ and $b \in \mathcal{B}^{\circ} \backslash \mathcal{B}$ in (3.15), we obtain the results.

Remark 3.11 The Cauchy type identity in Theorem 3.10 was also proved in a bijective way in terms of tableaux [27], which can be viewed as an analogue of the Robinson-Schensted-Knuth correspondence [26].

\subsection{Factorization property}

Let us consider a particular case, where we have a factorization property of $S_{\lambda}^{\mathcal{A} / \mathcal{B}}$. Recall the following Cauchy type identity for skew Schur functions.

Lemma 3.12 (cf. [29]) For $\lambda, \mu \in \mathcal{P}$, we have

$$
\sum_{\rho \in \mathcal{P}} s_{\rho / \lambda}(\mathbf{x}) s_{\rho / \mu}(\mathbf{y})=\frac{1}{\prod_{i, j \geq 1}\left(1-x_{i} y_{j}\right)} \sum_{\tau \in \mathcal{P}} s_{\mu / \tau}(\mathbf{x}) s_{\lambda / \tau}(\mathbf{y}),
$$

By similar arguments as in Theorem 3.10, it is straightforward to rewrite the above identities as follows.

Corollary 3.13 For $\lambda, \mu \in \mathcal{P}$, we have

$$
\sum_{\rho \in \mathcal{P}} S_{\rho / \lambda}^{\mathcal{A}} S_{\rho / \mu}^{\mathcal{B}}=\frac{\prod_{|a| \neq|b|}\left(1+x_{a} x_{b}\right)}{\prod_{|a|=|b|}\left(1-x_{a} x_{b}\right)} \sum_{\tau \in \mathcal{P}} S_{\mu / \tau}^{\mathcal{A}} S_{\lambda / \tau}^{\mathcal{B}}
$$


Lemma 3.14 Given $\lambda \in \mathbb{Z}_{+}^{d}$ and $m \in \mathbb{N}$, assume that $d \geq m$ and $\lambda_{m} \geq 0$. Let $p \geq 0$ and $\mu \in \mathcal{P}$ be such that $\lambda+\left(p^{d}\right) \in \mathcal{P}, \mu \subset \lambda+\left(p^{d}\right)$, and $\mu \subset\left(p^{d}\right)$. Then

$$
s_{\left(\lambda+\left(p^{d}\right)\right) / \mu}\left(\mathbf{x}_{[m]}\right)=s_{\lambda+}\left(\mathbf{x}_{[m]}\right) s_{v / \mu}\left(\mathbf{x}_{[m]}\right),
$$

where $v=\left(p^{d}\right)+\left(\lambda^{-}\right)^{*}$.

Proof Note that $s_{\rho / \tau}\left(\mathbf{x}_{[m]}\right)$ corresponding to a skew Young diagram $\rho / \tau$ is the weight generating function of $S S T_{[\mathrm{m}]}(\rho / \tau)$, the set of all semistandard tableaux of shape $\rho / \tau$ with entries in $[m]$ (cf. $[16,29])$.

Suppose that $S S T_{[m]}\left(\left(\lambda+\left(p^{d}\right)\right) / \mu\right)$ is not empty. As usual, we enumerate the rows (resp. columns) in $\lambda+\left(p^{d}\right)$ from top to bottom (resp. left to right). Also, we may assume that $\nu / \mu$ is not empty, where $\nu=\left(p^{d}\right)+\left(\lambda^{-}\right)^{*}$.

For $T \in S S T_{[m]}\left(\left(\lambda+\left(p^{d}\right)\right) / \mu\right)$, let $T_{1}$ (resp. $T_{2}$ ) be the subtableau obtained from the columns of $T$ with indices greater than $p$ (resp. less than or equal to $p$ ). The shapes of $T_{1}$ and $T_{2}$ are $\lambda^{+}$and $\nu / \mu$, respectively. So, this defines a map

$$
S S T_{[m]}\left(\left(\lambda+\left(p^{d}\right)\right) / \mu\right) \longrightarrow S S T_{[m]}\left(\lambda^{+}\right) \times S S T_{[m]}(\nu / \mu),
$$

by sending $T$ to $\left(T_{1}, T_{2}\right)$.

We claim that this is a one-to-one correspondence, which establishes the corresponding identity of Schur polynomials. Let us construct an inverse of the above map. Given a pair $\left(T_{1}, T_{2}\right) \in S S T_{[m]}\left(\lambda^{+}\right) \times S S T_{[m]}(\nu / \mu)$, we obtain a tableau $T$ (not necessarily semistandard) of shape $\left(\lambda+\left(p^{d}\right)\right) / \mu$, where the first $p$ columns form $T_{2}$, and the other columns form $T_{1}$. Note that the row and column numbers are those in $\lambda+\left(p^{d}\right)$.

For $1 \leq i \leq d$, let $a_{i}$ (resp. $b_{i}$ ) be the entry placed in the $i$ th row and the $p$ th (resp. $(p+1)$ th) column of $T$. We assume that either $a_{i}$ or $b_{i}$ is empty if there is no entry. Then $b_{1}, \ldots, b_{d}$ are the entries in the first column of $T_{1}$, and $a_{1}, \ldots, a_{d}$ are the entries in the last column of $T_{2}$.

Since $m \leq d, b_{m+1}, \ldots, b_{d}$ are empty, and for $1 \leq k \leq m, b_{k} \geq k$ if it is not empty. If all $a_{i}$ are empty, then it is clear that $T$ is semistandard. We assume that there exist non-empty entries $a_{s}, \ldots, a_{t}$ with $1 \leq s \leq t \leq d$. Since $\lambda_{m} \geq 0$, it follows that the $p$ th column of $\lambda+\left(p^{d}\right)$ has at least $m$ boxes, and hence $\left(\lambda+\left(p^{d}\right)\right) / \mu$ has at least one box in rows lower than or equal to the $m$ th row. This implies that $t \geq m$, and $a_{k} \leq m+k-t \leq k \leq b_{k}$ for $s \leq k \leq t$. Therefore, $T$ is a semistandard tableau of shape $\left(\lambda+\left(p^{d}\right)\right) / \mu$, and the map $\left(T_{1}, T_{2}\right) \mapsto T$ is the inverse of (3.16). This completes the proof.

Theorem 3.15 Suppose that $\mathcal{A}=[m]$ for $m>0$. For $\lambda \in \mathbb{Z}_{+}^{d}$, we have

$$
S_{\lambda}^{[m] / \mathcal{B}}=S_{\lambda+}\left(\mathbf{x}_{[m]}\right) S_{\lambda^{-}}\left(\mathbf{x}_{\mathcal{B}}^{-1}\right) \Delta_{[m] / \mathcal{B}}^{-1}
$$

if and only if $d \geq m$ and $\lambda_{m} \geq 0$. 
Proof First, note that by the Littlewood-Richardson rule, we have $S_{\mu}^{\mathcal{A}}=S_{\mu^{\pi}}^{\mathcal{A}}$ for any $\mathcal{A}$ and $\mu \in \mathcal{P}$, where $\mu^{\pi}$ is the skew Young diagram obtained from $\mu$ by $180^{\circ}$ rotation (cf. [29]). Now, suppose that $d \geq m$ and $\lambda_{m} \geq 0$. Then we have

$$
\begin{aligned}
& S_{\lambda}^{[m] / \mathcal{B}}=\sum_{\substack{\mu=\left(\lambda+\left(p^{d}\right)\right) / \eta \\
\nu=\left(p^{d}\right) / \eta}} s_{\mu}\left(\mathbf{x}_{[m]}\right) S_{\nu}\left(\mathbf{x}_{\mathcal{B}}^{-1}\right) \\
& =\sum_{\substack{\mu=\left(\lambda+\left(p^{d}\right)\right) / \eta \\
\nu=\left(p^{d}\right) / \eta}} s_{\lambda+}\left(\mathbf{x}_{[m]}\right) s_{\left(\left(p^{d}\right)+\left(\lambda^{-}\right)^{*}\right) / \eta}\left(\mathbf{x}_{[m]}\right) S_{\nu}\left(\mathbf{x}_{\mathcal{B}}^{-1}\right) \quad \text { (by Lemma 3.14) } \\
& =s_{\lambda+}\left(\mathbf{x}_{[m]}\right) \sum_{\substack{\nu=\left(p^{d}\right) / \eta \\
\lambda^{-} \subset v^{\pi}}} s_{v^{\pi} / \lambda^{-}}\left(\mathbf{x}_{[m]}\right) S_{v^{\pi}}\left(\mathbf{x}_{\mathcal{B}}^{-1}\right) \quad \text { (by } 180^{\circ} \text {-rotation) } \\
& =s_{\lambda+}\left(\mathbf{x}_{[m]}\right) \sum_{\lambda-\subset \tau} s_{\tau / \lambda-}\left(\mathbf{x}_{[m]}\right) S_{\tau}\left(\mathbf{x}_{\mathcal{B}}^{-1}\right) \\
& =S_{\lambda^{+}}\left(\mathbf{x}_{[m]}\right) S_{\lambda^{-}}\left(\mathbf{x}_{\mathcal{B}}^{-1}\right) \Delta_{[m] / \mathcal{B}}^{-1} \quad \text { (by Corollary 3.13). }
\end{aligned}
$$

Conversely, suppose that either $d<m$ or $\lambda_{m}<0$. Let $p \geq 0$ and $\eta \in \mathcal{P}$ be such that $\lambda+\left(p^{d}\right) \in \mathcal{P}$ and $\eta \subset \lambda+\left(p^{d}\right), \eta \subset\left(p^{d}\right)$. Then it is not difficult to see that the difference

$$
s_{\lambda+}\left(\mathbf{x}_{[m]}\right) s_{\left(\left(p^{d}\right)+\left(\lambda^{-}\right)^{*}\right) / \eta}\left(\mathbf{x}_{[m]}\right)-s_{\left(\lambda+\left(p^{d}\right)\right) / \eta}\left(\mathbf{x}_{[m]}\right) .
$$

is non-zero, in fact, an integral linear combination of monomials in $\mathbf{x}_{[m]}$ with nonnegative coefficients. It implies that the difference of $S_{\lambda}^{[m] / \mathcal{B}}$ and the last term in (3.17) is non-zero. This completes the proof.

Remark 3.16 We can also prove Theorem 3.15 using Theorem 3.10. Suppose that $\lambda \in \mathbb{Z}_{+}^{d}$ is given. If $d \geq m$ and $\lambda_{m} \geq 0$, then it is not difficult to see that $\ell\left(\lambda^{w,+}\right) \leq m$ if and only if $w=1 \in \mathcal{W}$, which implies that $S_{\lambda}^{[m] / \mathcal{B}}=S_{\lambda+}\left(\mathbf{x}_{[m]}\right) S_{\lambda^{-}}\left(\mathbf{x}_{\mathcal{B}}^{-1}\right) \Delta_{[m] / \mathcal{B}}^{-1}$. Conversely, if $d<m$ or $\lambda_{m}<0$, then we can check that there exists at least one nontrivial element $w \in \mathcal{W}$ such that $\ell\left(\lambda^{w,+}\right) \leq m$, which implies that $S_{\lambda}^{[m] / \mathcal{B}} \Delta_{[m] / \mathcal{B}}-$ $S_{\lambda+}\left(\mathbf{x}_{[m]}\right) S_{\lambda^{-}}\left(\mathbf{x}_{\mathcal{B}}^{-1}\right)$ is non-zero by Remark 3.3 (2).

\subsection{Jacobi-Trudi formula}

Finally, let us present another proof of the Jacobi-Trudi formula for $S_{\lambda}^{\mathcal{A} / \mathcal{B}}$ [27] using the arguments given in 3.1.

Given $\mathcal{A}$ and $\mathcal{B}$, consider

$$
H^{\mathcal{A} / \mathcal{B}}(t)=\frac{\prod_{a \in \mathcal{A}_{1}}\left(1+x_{a} t\right) \prod_{b \in \mathcal{B}_{1}}\left(1+x_{b}^{-1} t^{-1}\right)}{\prod_{a \in \mathcal{A}_{0}}\left(1-x_{a} t\right) \prod_{b \in \mathcal{B}_{0}}\left(1-x_{b}^{-1} t^{-1}\right)}=\sum_{k \in \mathbb{Z}} h_{k}^{\mathcal{A} / \mathcal{B}} t^{k},
$$

where $h_{k}^{\mathcal{A} / \mathcal{B}}=S_{k}^{\mathcal{A} / \mathcal{B}}=\sum_{m-n=k} S_{m}\left(\mathbf{x}_{\mathcal{A}}\right) S_{n}\left(\mathbf{x}_{\mathcal{B}}^{-1}\right)$ for $k \in \mathbb{Z}$. 
Proposition 3.17 cf. [27] For $\lambda \in \mathbb{Z}_{+}^{d}$, we have

$$
S_{\lambda}^{\mathcal{A} / \mathcal{B}}=\operatorname{det}\left(h_{\lambda_{i}-i+j}^{\mathcal{A} / \mathcal{B}}\right)_{1 \leq i, j \leq d}
$$

Proof Choose $p, q>0$. Let $e_{k}\left(\mathbf{x}_{[-p, q]^{\prime}}\right)$ be the $k$ th elementary symmetric polynomial in variables $\mathbf{x}_{[-p, q]^{\prime}}$ for $k \geq 0$, and $\widetilde{e}_{k}\left(\mathbf{x}_{[-p, q]^{\prime}}\right)$ the coefficient of $t^{k}$ for $-p \leq k \leq q$ in

$$
\prod_{i \in[-p]^{\prime}} \prod_{j \in[q]^{\prime}}\left(1+x_{i}^{-1} t^{-1}\right)\left(1+x_{j} t\right) .
$$

Then, from the classical Jacobi-Trudi formula, we have

$$
\begin{aligned}
S_{\lambda}^{[q]^{\prime} /[-p]^{\prime}} & =\left(x_{-p^{\prime}} \cdots x_{-1^{\prime}}\right)^{-d} s_{\left(\lambda+\left(p^{d}\right)\right)^{\prime}}\left(\mathbf{x}_{[-p, q]^{\prime}}\right) \\
& =\left(x_{-p^{\prime}} \cdots x_{-1^{\prime}}\right)^{-d} \operatorname{det}\left(e_{\lambda_{i}+p-i+j}\left(\mathbf{x}_{[-p, q]^{\prime}}\right)\right)_{1 \leq i, j \leq d} \\
& =\operatorname{det}\left(\widetilde{e}_{\lambda_{i}-i+j}\left(\mathbf{x}_{[-p, q]^{\prime}}\right)\right)_{1 \leq i, j \leq d} .
\end{aligned}
$$

If we follow the same arguments as in Theorem 3.10, then $S_{\lambda}^{[q]^{\prime} /[-p]^{\prime}}$ and $\widetilde{e}_{k}\left(\mathbf{x}_{[-p, q]^{\prime}}\right)$ are replaced by $S_{\lambda}^{\mathcal{A} / \mathcal{B}}$ and $h_{k}^{\mathcal{A} / \mathcal{B}}$, respectively. This completes the proof.

\section{Applications}

In this section, we discuss applications of our Weyl type formula for $S_{\lambda}^{\mathcal{A} / \mathcal{B}}$ in representation theory. We assume that the ground field is $\mathbb{C}$.

\subsection{Representations of $\mathfrak{g l}_{m \mid n}$}

First, let us derive a Weyl type formula for hook Schur polynomials. Though its proof is purely combinatorial, we will translate the result in the language of representation theory to see its relation with finite dimensional irreducible representations of $\mathfrak{g l}_{m \mid n}$. So, let us give a brief review on representations of the Lie superalgebra $\mathfrak{g l}_{m \mid n}$ (cf. [20]).

For non-negative integers $m$ and $n$, not both zero, let $\mathbb{C}^{m \mid n}=\mathbb{C}^{m \mid 0} \oplus \mathbb{C}^{0 \mid n}$ be the $(m+n)$-dimensional superspace with the even subspace $\mathbb{C}^{m \mid 0}=\mathbb{C}^{m}$ and the odd subspace $\mathbb{C}^{0 \mid n}=\mathbb{C}^{n}$. We denote by $\left\{\epsilon_{i} \mid i \in[-m]\right\}$ and $\left\{\epsilon_{j} \mid j \in[n]^{\prime}\right\}$ the homogeneous bases of $\mathbb{C}^{m \mid 0}$ and $\mathbb{C}^{0 \mid n}$ respectively, which form a standard basis of $\mathbb{C}^{m \mid n}$. Then the space of $\mathbb{C}$-linear endomorphisms of $\mathbb{C}^{m \mid n}$ is naturally equipped with a $\mathbb{Z}_{2}$-grading, and becomes a Lie superalgebra with respect to a super bracket, which is called a general linear superalgebra $\mathfrak{g l}_{m \mid n}$. Put $\left[-m, n^{\prime}\right]=[-m] \cup[n]^{\prime}$.

We may identify $\mathfrak{g}=\mathfrak{g l}_{m \mid n}$ with the set of $(m+n) \times(m+n)$ matrices with respect to the standard basis of $\mathbb{C}^{m \mid n}$. Then the subspace $\mathfrak{h}$ of diagonal matrices forms a Cartan subalgebra, and under the adjoint action of $\mathfrak{h}$ on $\mathfrak{g}$, we have a root space decomposition, $\mathfrak{g}=\mathfrak{h} \oplus\left(\bigoplus_{\alpha \in \Delta} \mathfrak{g}_{\alpha}\right)$, where $\Delta$ is the set of all roots of $\mathfrak{g}$. Let $\Delta^{+}$be 
the set of positive roots, and $\Delta_{0}^{+}$(resp. $\Delta_{1}^{+}$) the set of positive even (resp. odd) roots. Since $\mathfrak{h}^{*}=\mathbb{C}^{m \mid n}$, we have

$$
\begin{aligned}
& \Delta_{0}^{+}=\left\{\epsilon_{i}-\epsilon_{j}\left|i, j \in\left[-m, n^{\prime}\right],\right| i|=| j \mid, i<j\right\}, \\
& \Delta_{1}^{+}=\left\{\epsilon_{i}-\epsilon_{j} \mid i \in[-m], j \in[n]^{\prime}\right\},
\end{aligned}
$$

where we assume that $-m<-m+1<\cdots<-1<1^{\prime}<2^{\prime}<\cdots<n^{\prime}$. We consider a $\mathbb{Z}$-grading $\mathfrak{g}=\mathfrak{g}_{-1} \oplus \mathfrak{g}_{0} \oplus \mathfrak{g}_{1}$ consistent with its parity, where $\mathfrak{g}_{0}=\mathfrak{g l}_{m} \oplus \mathfrak{g l}_{n}$, and a triangular decomposition, $\mathfrak{g}=\mathfrak{n}^{-} \oplus \mathfrak{h} \oplus \mathfrak{n}^{+}$such that $\mathfrak{g}_{ \pm 1} \subset \mathfrak{n}^{ \pm}$.

Let $P_{m \mid n}$ be the $\mathbb{Z}$-lattice of $\mathfrak{h}^{*}$ generated by $\left\{\epsilon_{i} \mid i \in\left[-m, n^{\prime}\right]\right\}$, which is called the set of integral weights, and let $P_{m \mid n}^{+}$be the set of weights $\Lambda=\sum_{i \in\left[-m, n^{\prime}\right]} \Lambda_{i} \epsilon_{i} \in P_{m \mid n}$ such that $\Lambda_{-m} \geq \ldots \geq \Lambda_{-1}$ and $\Lambda_{1^{\prime}} \geq \ldots \geq \Lambda_{n^{\prime}}$. An element in $P_{m \mid n}^{+}$is called a dominant integral weight.

Suppose that $M$ is a finite-dimensional $\mathfrak{g l}_{m \mid n}$-module, which is $\mathfrak{h}$-diagonalizable. Then, we have a weight space decomposition $M=\bigoplus_{\lambda \in \mathfrak{h}^{*}} M_{\lambda}$. When $\operatorname{dim} M_{\lambda} \neq 0$, we call $\lambda$ weights of $M$. For convenience, we assume that all the weight are integral. We define the character of $M$ by $\operatorname{ch} M=\sum_{\lambda \in P_{m \mid n}} \operatorname{dim} M_{\lambda} e^{\lambda}$, where $\left\{e^{\lambda} \mid \lambda \in P_{m \mid n}\right\}$ is the set of formal variables.

Given $\Lambda \in P_{m \mid n}^{+}$, let $L^{0}(\Lambda)$ be the finite dimensional irreducible highest weight $\mathfrak{g}_{0}$-module with highest weight $\Lambda$. We may view $L^{0}(\Lambda)$ as a $\mathfrak{g}_{0} \oplus \mathfrak{g}_{1}$-module, where $\mathfrak{g}_{1}$ acts trivially on $L^{0}(\Lambda)$. The Kac module $K_{m \mid n}(\Lambda)$ is defined to be the induced representation $K_{m \mid n}(\Lambda)=U(\mathfrak{g}) \otimes_{U\left(\mathfrak{g}_{0} \oplus \mathfrak{g}_{1}\right)} L^{0}(\Lambda)$ where $U(\mathfrak{g})$ and $U\left(\mathfrak{g}_{0} \oplus \mathfrak{g}_{1}\right)$ are the enveloping algebras, and it has a unique maximal irreducible quotient $L_{m \mid n}(\Lambda)$. Then $\left\{L_{m \mid n}(\Lambda) \mid \Lambda \in P_{m \mid n}^{+}\right\}$forms a complete set of pairwise non-isomorphic finite dimensional irreducible representations of $\mathfrak{g l}_{m \mid n}$ with integral weights.

For $\Lambda \in P_{m \mid n}^{+}$such that $K_{m \mid n}(\Lambda)$ is not irreducible (cf. [21, 22]), $L_{m \mid n}(\Lambda)$ has a resolution, where each term has a filtration with quotients isomorphic to Kac modules (see [30]), and its character is given by

$$
\operatorname{ch} L_{m \mid n}(\Lambda)=\sum_{\Lambda^{\prime} \in P_{m \mid n}^{+}} a_{\Lambda \Lambda^{\prime}} \operatorname{ch} K_{m \mid n}\left(\Lambda^{\prime}\right),
$$

for some $a_{\Lambda \Lambda^{\prime}} \in \mathbb{Z}$. In [30], Serganova gave an algorithm for computing these coefficients using the geometry of the associated supergroups. Recently, in [5], Brundan gave another algorithm using a remarkable connection with canonical bases of quantum group $U_{q}\left(\mathfrak{g l}_{\infty}\right)$.

Now, let us give an explicit expression of (4.2) for irreducible tensor representations. For $\Lambda=\sum_{i \in\left[-m, n^{\prime}\right]} \Lambda_{i} \epsilon_{i} \in P_{m \mid n}^{+}$, we may identify $\Lambda$ with a pair of generalized partitions given by

$$
\Lambda^{<0}=\left(\Lambda_{-m}, \ldots, \Lambda_{-1}\right) \in \mathbb{Z}_{+}^{m}, \quad \Lambda^{>0}=\left(\Lambda_{1^{\prime}}, \ldots, \Lambda_{n^{\prime}}\right) \in \mathbb{Z}_{+}^{n}
$$


If we put $\mathbf{x}_{[-m]}=\left\{x_{i}=e^{\epsilon_{i}} \mid i \in[-m]\right\}$, and $\mathbf{y}_{[n]}=\left\{y_{j}=e^{\epsilon_{j^{\prime}}} \mid j \in[n]\right\}$, then the character of $K_{m \mid n}(\Lambda)$ is given by

$$
\operatorname{ch} K_{m \mid n}(\Lambda)=s_{\Lambda<0}\left(\mathbf{x}_{[-m]}\right) s_{\Lambda^{>0}}\left(\mathbf{y}_{[n]}\right) \prod_{\substack{i \in[-m], j \in[n]}}\left(1+x_{i}^{-1} y_{j}\right)
$$

Let $\widehat{P}_{m \mid n}^{+}$be the set of dominant integral weights $\Lambda$ such that $\Lambda_{k} \geq 0$ for all $k \in$ $\left[-m, n^{\prime}\right]$ and $\ell\left(\left(\Lambda^{>0}\right)^{\prime}\right) \leq \Lambda_{-1}$. For $\Lambda \in \widehat{P}_{m \mid n}^{+}$, we define a partition

$$
\lambda(\Lambda)=\left(\Lambda_{-m}, \ldots, \Lambda_{-1}, \Lambda_{1}^{\prime}, \ldots, \Lambda_{\ell}^{\prime}\right),
$$

where $\left(\Lambda^{>0}\right)^{\prime}=\left(\Lambda_{1}^{\prime}, \ldots, \Lambda_{\ell}^{\prime}\right)$ and $\ell=\ell\left(\left(\Lambda^{>0}\right)^{\prime}\right)$. Then the map $\Lambda \mapsto \lambda(\Lambda)$ gives a one-to-one correspondence between $\widehat{P}_{m \mid n}^{+}$and $\mathcal{P}_{m \mid n}=\left\{\lambda \in \mathcal{P} \mid \lambda_{m+1} \leq n\right\}$, the set of all $(m, n)$-hook partitions.

In $[3,31]$, it was shown that the tensor algebra $\mathcal{T}\left(\mathbb{C}^{m \mid n}\right)$ generated by the natural representation $\mathbb{C}^{m \mid n}$ is completely reducible, and for $\Lambda \in P_{m \mid n}^{+}, L_{m \mid n}(\Lambda)$ occurs in $\mathcal{T}\left(\mathbb{C}^{m \mid n}\right)$ if and only if $\Lambda \in \widehat{P}_{m \mid n}^{+}$. Moreover, we have

$$
\operatorname{ch} L_{m \mid n}(\Lambda)=\sum_{\mu \subset \lambda} s_{\mu}\left(\mathbf{x}_{[-m]}\right) s_{\lambda^{\prime} / \mu^{\prime}}\left(\mathbf{y}_{[n]}\right)
$$

with $\lambda=\lambda(\Lambda) \in \mathscr{P}_{m \mid n}$, which is called the hook Schur polynomial corresponding to $\lambda$. Following our notation, $\operatorname{ch} L_{m \mid n}(\Lambda)=S_{\lambda}^{\left[-m, n^{\prime}\right]}=S_{\lambda}\left(\mathbf{x}_{\left[-m, n^{\prime}\right]}\right)$, where we identify $\mathbf{x}_{[n]^{\prime}} \subset \mathbf{x}_{\left[-m, n^{\prime}\right]}$ with $\mathbf{y}_{[n]}$.

Lemma 4.1 For $\Lambda \in \widehat{P}_{m \mid n}^{+}$, let $\lambda=\lambda(\Lambda) \in \mathcal{P}_{m \mid n}$ and $v=\lambda^{\prime}-\left(m^{d}\right)$ for $d \geq \lambda_{1}$. Then $v \in \mathbb{Z}_{+}^{d}$, and

$$
\left(x_{-m} \cdots x_{-1}\right)^{-d} \operatorname{ch} L_{m \mid n}(\Lambda)=S_{v}\left(\mathbf{x}_{[n]} ; \mathbf{x}_{[-m]^{\prime}}\right),
$$

where we identify $\mathbf{x}_{[-m]}$ with $\mathbf{x}_{[-m]^{\prime}}$, and $\mathbf{y}_{[n]}$ with $\mathbf{x}_{[n]}$.

Proof By similar arguments as in Lemma 3.5 (3.10), we have

$$
\begin{aligned}
& \left(x_{-m} \cdots x_{-1}\right)^{-d} \operatorname{ch} L_{m \mid n}(\Lambda) \\
& =\left(x_{-m} \cdots x_{-1}\right)^{-d}\left(\sum_{\mu \subset \lambda} s_{\mu}\left(\mathbf{x}_{[-m]}\right) s_{\lambda^{\prime} / \mu^{\prime}}\left(\mathbf{y}_{[n]}\right)\right) \\
& =\sum_{\mu \subset \lambda} s_{\mu-\left(d^{m}\right)}\left(\mathbf{x}_{[-m]}\right) s_{\lambda^{\prime} / \mu^{\prime}}\left(\mathbf{y}_{[n]}\right) \\
& =\sum_{\mu \subset \lambda} s_{\left(d^{m}\right) / \mu}\left(\mathbf{x}_{[-m]}^{-1}\right) s_{\lambda^{\prime} / \mu^{\prime}}\left(\mathbf{y}_{[n]}\right)
\end{aligned}
$$




$$
\begin{aligned}
& =\sum_{\eta} S_{\left(m^{d}\right) / \eta}\left(\mathbf{x}_{[-m]^{\prime}}^{-1}\right) S_{\left(v+\left(m^{d}\right)\right) / \eta}\left(\mathbf{y}_{[n]}\right) \quad \text { by replacing } \mathbf{x}_{[-m]} \text { with } \mathbf{x}_{[-m]^{\prime}} \\
& =S_{v}\left(\mathbf{x}_{[n]} ; \mathbf{x}_{[-m]^{\prime}}\right)=S_{v}^{[n] /[-m]^{\prime}} \quad \text { by replacing } \mathbf{y}_{[n]} \text { with } \mathbf{x}_{[n]} .
\end{aligned}
$$

Remark 4.2 It is well-known that for $\Lambda \in \widehat{P}_{m \mid n}^{+}, L_{m \mid n}(\Lambda)=K_{m \mid n}(\Lambda)$ if and only if $\Lambda_{-1} \geq n$ (see also Theorem 3.15).

For $\Lambda \in \widehat{P}_{m \mid n}^{+}$with $\lambda=\lambda(\Lambda)$, set

$$
\mathcal{W}_{m \mid n}(\Lambda)=\left\{w \in \mathcal{W} \mid \ell\left(\left(v^{w,-}\right)^{\prime}\right) \leq m, \ell\left(v^{w,+}\right) \leq n\right\},
$$

where $v=\lambda^{\prime}-\left(m^{d}\right) \in \mathbb{Z}_{+}^{d}$ with $d \geq \lambda_{1}$. For $w \in \mathcal{W}_{m \mid n}(\Lambda)$, we define

$$
w * \Lambda=\left(\left(\left(v^{w,-}\right)^{\prime}-\left(d^{m}\right)\right)^{*}, v^{w,+}\right) \in P_{m \mid n}^{+},
$$

where we identify $P_{m \mid n}^{+}$with $\mathbb{Z}_{+}^{m} \times \mathbb{Z}_{+}^{n}$ (cf.(4.3)). We can check that given $w \in$ $\mathcal{W}_{m \mid n}(\Lambda), w \in W_{I(m, q)}$ for some $q$, and $w * \Lambda$ does not depend on the choice of $d$. Now, we obtain a Weyl type formula for hook Schur polynomials, which recovers the Cheng and Zhang's formula [10] in a new combinatorial way.

Theorem 4.3 ([10]) For $\Lambda \in \widehat{P}_{m \mid n}^{+}$, we have

$$
\operatorname{ch} L_{m \mid n}(\Lambda)=\sum_{w \in \mathcal{W}_{m \mid n}(\Lambda)}(-1)^{\ell(w)} \operatorname{ch} K_{m \mid n}(w * \Lambda) .
$$

Proof By Lemma 4.1 and Theorem 3.10, we have

$$
\begin{aligned}
\operatorname{ch} & L_{m \mid n}(\Lambda) \\
& =\left(x_{-m} \cdots x_{-1}\right)^{d}\left(\sum_{w \in \mathcal{W}}(-1)^{\ell(w)} s_{v^{w,+}}\left(\mathbf{y}_{[n]}\right) s_{\left(v^{w,-}\right)^{\prime}}\left(\mathbf{x}_{[-m]}^{-1}\right)\right) \prod_{\substack{i \in[-m] \\
j \in[n]}}\left(1+x_{i}^{-1} y_{j}\right) \\
& =\left(\sum_{w \in \mathcal{W}}(-1)^{\ell(w)} s_{v^{w,+}}\left(\mathbf{y}_{[n]}\right) s_{\left(v^{w,-}\right)^{\prime}-\left(d^{m}\right)}\left(\mathbf{x}_{[-m]}^{-1}\right)\right) \prod_{\substack{i \in[-m] \\
j \in[n]}}\left(1+x_{i}^{-1} y_{j}\right) \\
& =\left(\sum_{w \in \mathcal{W}}(-1)^{\ell(w)} s_{v^{w,+}}\left(\mathbf{y}_{[n]}\right) s_{\left.\left(\left(v^{w,-}\right)^{\prime}-\left(d^{m}\right)\right)^{*}\left(\mathbf{x}_{[-m]}\right)\right)} \prod_{\substack{i \in[-m] \\
j \in[n]}}\left(1+x_{i}^{-1} y_{j}\right)\right. \\
& =\sum_{w \in \mathcal{W}_{m \mid n}(\Lambda)}(-1)^{\ell(w)} \operatorname{ch} K(w * \Lambda)
\end{aligned}
$$

Remark 4.4 Lemma 4.1 and hence Theorem 4.3 can be also derived from a Howe duality of $\left(\mathfrak{g l}_{m \mid n}, \mathfrak{g l}_{d}\right)$ acting on the supersymmetric algebra generated by $\mathbb{C}^{m \mid n} \otimes \mathbb{C}^{d}$ 
(cf. [7]) without using the combinatorial formula for $\operatorname{ch} L_{m} \mid n(\Lambda)$ due to Berele and Regev.

The denominator identity for $\mathfrak{g l}_{m \mid n}$ is given as follows.

Corollary 4.5 For $w \in \mathcal{W}_{m \mid n}(0)$, let $w * 0=\left(\theta_{m}^{w}, \theta_{n}^{w}\right) \in P_{m \mid n}^{+}$. Then

$$
\begin{aligned}
\prod_{i, j}\left(x_{i}+y_{j}\right)^{-1} \prod_{i<k}\left(x_{i}-x_{k}\right) \prod_{j<l}\left(y_{j}-y_{l}\right) \\
=\sum_{w \in \mathcal{W}_{m \mid n}(0)} \sum_{\substack{w_{1} \in S_{m} \\
w_{2} \in S_{n}}}(-1)^{\ell\left(w_{1}\right)+\ell\left(w_{2}\right)+\ell(w)} \mathbf{x}_{[-m]}^{w_{1}\left(\theta_{m}^{w}+\rho_{m}^{-}\right)-n \mathbf{1}_{m}} \mathbf{y}_{[n]}^{w_{2}\left(\theta_{n}^{w}+\rho_{n}^{+}\right)},
\end{aligned}
$$

where $i, k \in[-m], j, l \in[n], \rho_{m}^{-}=\sum_{i \in[-m]}(-i-1) \epsilon_{i}, \rho_{n}^{+}=\sum_{j \in[n]}(n-j) \epsilon_{j^{\prime}}$, and $S_{m}$ (resp. $\left.S_{n}\right)$ is the symmetric group on the letters $[-m]$ (resp. $\left.[n]\right)$.

Proof Since the hook Schur polynomial corresponding to empty partition (that is, $\Lambda=0$ ) is 1 , the identity follows from Theorem 4.3.

Remark 4.6 For $w \in \mathcal{W}_{m \mid n}(0)$, let $\mu$ be the corresponding partition (see Remark 3.3). Then $w * 0 \in P_{m \mid n}^{+}$if and only if $\ell(\mu) \leq m, n$. Moreover, in this case, we have

$$
w * 0=\left(\theta_{m}^{w}, \theta_{n}^{w}\right)=\left(-\mu_{m}, \ldots,-\mu_{1}, \mu_{1}, \ldots, \mu_{n}\right) \in P_{m \mid n}^{+} .
$$

Since $|\mu|=\ell(w)$, we may write by abuse of notation

$$
\begin{aligned}
\prod_{i, j}\left(x_{i}+y_{j}\right)^{-1} \prod_{i<k}\left(x_{i}-x_{k}\right) \prod_{j<l}\left(y_{j}-y_{l}\right) \\
=\sum_{\ell(\mu) \leq m, n}(-1)^{|\mu|} \sum_{\substack{w_{1} \in S_{m} \\
w_{2} \in S_{n}}}(-1)^{\ell\left(w_{1}\right)+\ell\left(w_{2}\right)} \mathbf{x}_{[-m]}^{w_{1}\left(\mu^{*}+\rho_{m}^{-}\right)-n \mathbf{1}_{m}} \mathbf{y}_{[n]}^{w_{2}\left(\mu+\rho_{n}^{+}\right)} .
\end{aligned}
$$

\subsection{Representations of $\mathfrak{g l}_{m+n}$}

Let us consider a character formula for a certain class of infinite dimensional irreducible highest weight representations of the Lie algebra $\mathfrak{g l}_{m+n}$, which comes from the study of unitary highest weight representations of its associated Lie group $U(m, n)($ cf. $[15,19,24])$ or from a parabolic analogue of Kazhdan-Lusztig theory (cf. $[6,13])$.

For non-negative integers $m$ and $n$, not both zero, let $\mathbb{C}^{m+n}=\mathbb{C}^{m} \oplus \mathbb{C}^{n}$ be the $(m+n)$-dimensional space, where $\left\{\epsilon_{i} \mid i \in[-m]\right\}$ (resp. $\left.\left\{\epsilon_{j} \mid j \in[n]\right\}\right)$ is the basis of $\mathbb{C}^{m}$ (resp. $\mathbb{C}^{n}$ ). We may identify the general linear algebra $\mathfrak{g}=\mathfrak{g l}_{m+n}$ with the set of $(m+n) \times(m+n)$ matrices whose row and column indices are from $[-m] \cup[n]$ (or simply $[-m, n]$ ).

We denote by $\mathfrak{h}$ and $\mathfrak{b}$ the Cartan subalgebra of the diagonal matrices and the Borel subalgebra of the upper triangular matrices respectively. We put $\mathfrak{t}=\mathfrak{g l}_{m} \oplus \mathfrak{g l}_{n}$, which 
is naturally embedded in $\mathfrak{g}$. Let $P_{m+n}$ be the $\mathbb{Z}$-lattice of $\mathfrak{h}^{*}$ generated by $\left\{\epsilon_{i} \mid i \in\right.$ $[-m, n]\}$, and $P_{m+n}^{+}$the set of $\mathfrak{t}$-dominant integral weights, that is, the set of weights $\Lambda=\sum_{i \in[-m, n]} \Lambda_{i} \epsilon_{i} \in P_{m+n}$ such that $\Lambda_{-m} \geq \ldots \geq \Lambda_{-1}$ and $\Lambda_{1} \geq \ldots \geq \Lambda_{n}$.

Given $\Lambda \in P_{m+n}^{+}$, let $L^{0}(\Lambda)$ be the finite dimensional irreducible highest weight t-module with highest weight $\Lambda$. We may view $L^{0}(\Lambda)$ as a representation of the parabolic subalgebra $\mathfrak{q}=\mathfrak{t}+\mathfrak{b}$, where the nilpotent radical of $\mathfrak{q}$ acts trivially. Now we define the generalized Verma module $V_{m+n}(\Lambda)$ to be the induced representation $V_{m+n}(\Lambda)=U(\mathfrak{g}) \otimes_{U(\mathfrak{q})} L^{0}(\Lambda)$. Then $V_{m+n}(\Lambda)$ has a unique maximal irreducible quotient $L_{m+n}(\Lambda)$. Similarly, we define the characters of $L_{m+n}(\Lambda)$ and $V_{m+n}(\Lambda)$ in terms of the formal variables $e^{\lambda}\left(\lambda \in P_{m+n}\right)$. Put $x_{i}=e^{\epsilon_{i}}$ for $i \in[-m, n]$. Then

$$
\operatorname{ch} V_{m+n}(\Lambda)=\frac{s_{\Lambda<0}\left(\mathbf{x}_{[-m]}\right) s_{\Lambda>0}\left(\mathbf{x}_{[n]}\right)}{\prod_{i, j}\left(1-x_{i}^{-1} x_{j}\right)}
$$

where $i \in[-m], j \in[n], \Lambda^{<0}=\left(\Lambda_{-m}, \ldots, \Lambda_{-1}\right)$ and $\Lambda^{>0}=\left(\Lambda_{1}, \ldots, \Lambda_{n}\right)$.

From the Kazhdan-Lusztig conjecture [25] proved in [1,2] and its parabolic analogue $[6,13]$, we have

$$
\operatorname{ch} L_{m+n}(\Lambda)=\sum_{\Lambda^{\prime} \in P_{m+n}^{+}} b_{\Lambda \Lambda^{\prime}} \operatorname{ch} V_{m+n}\left(\Lambda^{\prime}\right),
$$

where the integers $b_{\Lambda \Lambda^{\prime}}$ are determined explicitly in terms of (parabolic) KazhdanLusztig polynomials evaluated at 1 .

Now, using Howe duality let us derive an explicit expression of $\operatorname{ch} L_{m+n}(\Lambda)$ for particular highest weights in $P_{m+n}^{+}$, which is equivalent to the Enright's formula given in $[12,14]$. For $d \geq 1$, let $P_{d}=\bigoplus_{k \in[d]} \mathbb{Z} \varepsilon_{k}$ be the weight lattice of $\mathfrak{g l}_{d}$. Consider the symmetric algebra $\varsigma_{d}$ generated by $\left(\mathbb{C}^{m^{*}} \otimes \mathbb{C}^{d^{*}}\right) \oplus\left(\mathbb{C}^{n} \otimes \mathbb{C}^{d}\right)$, where $\mathbb{C}^{m^{*}}$ and $\mathbb{C}^{d^{*}}$ are the duals of the natural representations of $\mathfrak{g l}_{m}$ and $\mathfrak{g l}_{d}$ respectively. Then there is a semi-simple $\left(\mathfrak{g l}_{m+n}, \mathfrak{g l}_{d}\right)$-action on $\S_{d}$, which gives the following multiplicity free decomposition $[19,24]$

$$
s_{d}=\bigoplus_{\lambda} L_{m+n}(\Lambda(\lambda)) \otimes L_{d}(\lambda)
$$

where the sum ranges over $\lambda \in \mathbb{Z}_{+}^{d}$ with $\ell\left(\lambda^{-}\right) \leq m$ and $\ell\left(\lambda^{+}\right) \leq n, L_{d}(\lambda)$ is the irreducible highest weight $\mathfrak{g l}_{d}$-module with highest weight $\lambda=\left(\lambda_{1}, \ldots, \lambda_{d}\right) \in \mathbb{Z}_{+}^{d}$ (or $\lambda=\sum_{k \in[d]} \lambda_{k} \varepsilon_{k} \in P_{d}$ ), and $\Lambda(\lambda)$ is a highest weight in $P_{m+n}^{+}$. In fact, if

$$
\lambda=\lambda^{+}+\left(\lambda^{-}\right)^{*}=\left(\lambda_{1}^{+}, \ldots, \lambda_{p}^{+}, 0, \ldots, 0,-\lambda_{q}^{-}, \ldots,-\lambda_{1}^{-}\right),
$$

with $q \leq m$ and $p \leq n$, then

$$
\Lambda(\lambda)=\underbrace{\left(-d, \ldots,-d,-\lambda_{q}^{-}-d, \ldots,-\lambda_{1}^{-}-d\right.}_{m}, \underbrace{\lambda_{1}^{+}, \ldots, \lambda_{p}^{+}, 0, \ldots, 0}_{n}),
$$

where we identify $P_{m+n}$ with $\mathbb{Z}^{m+n}$. 
Proposition 4.7 For $\lambda \in \mathbb{Z}_{+}^{d}$ with $\ell\left(\lambda^{-}\right) \leq m$ and $\ell\left(\lambda^{+}\right) \leq n$, we have

$$
\left(x_{-m} \cdots x_{-1}\right)^{d} \operatorname{ch} L_{m+n}(\Lambda(\lambda))=S_{\lambda}\left(\mathbf{x}_{[n]} ; \mathbf{x}_{[-m]}\right) \text {. }
$$

Proof The decomposition in (4.10) gives the following identity

$$
\frac{\left(x_{-m} \cdots x_{-1}\right)^{-d}}{\prod_{i, j, k}\left(1-x_{i} z_{k}\right)\left(1-x_{j}^{-1} z_{k}^{-1}\right)}=\sum_{\lambda \in \mathbb{Z}_{+}^{d}} \operatorname{ch} L_{m+n}(\Lambda(\lambda)) s_{\lambda}\left(\mathbf{z}_{[d]}\right),
$$

where $i \in[n], j \in[-m], k \in[d]$, and $\mathbf{z}_{[d]}=\left\{z_{k}=e^{\varepsilon_{k}} \mid k \in[d]\right\}$. Comparing with the Cauchy type identity in Theorem 3.10, it follows from the linear independence of rational Schur polynomials that $\left(x_{-m} \cdots x_{-1}\right)^{d} \operatorname{ch} L_{m+n}(\Lambda(\lambda))=S_{\lambda}^{[n] /[-m]}$.

Corollary 4.8 For $\lambda \in \mathbb{Z}_{+}^{d}$ with $\ell\left(\lambda^{-}\right) \leq m$ and $\ell\left(\lambda^{+}\right) \leq n$, we have $L_{m+n}(\Lambda(\lambda))=$ $V_{m+n}(\Lambda(\lambda))$ if and only if $d \geq n$ and $\lambda_{n} \geq 0$.

Proof It follows from Theorem 3.15.

We also have an interesting analogue of the Jacobi-Trudi formula from Proposition 3.17 .

Corollary 4.9 For $\lambda \in \mathbb{Z}_{+}^{d}$ with $\ell\left(\lambda^{-}\right) \leq m$ and $\ell\left(\lambda^{+}\right) \leq n$, we have

$$
\operatorname{ch} L_{m+n}(\Lambda(\lambda))=\operatorname{det}\left(\operatorname{ch} L_{m+n}\left(\Lambda\left(\lambda_{i}-i+j\right)\right)\right)_{1 \leq i, j \leq d} .
$$

Suppose that $\Lambda \in P_{m+n}^{+}$is given and $\Lambda=\Lambda(\lambda)$ for some $\lambda \in \mathbb{Z}_{+}^{d}$. Set

$$
\mathcal{W}_{m+n}(\Lambda)=\left\{w \in \mathcal{W} \mid \ell\left(\lambda^{w,-}\right) \leq m, \ell\left(\lambda^{w,+}\right) \leq n\right\} .
$$

For $w \in \mathcal{W}_{m+n}(\Lambda)$, we define

$$
w * \Lambda=\left(\left(\lambda^{w,-}+\left(d^{m}\right)\right)^{*}, \lambda^{w,+}\right) \in P_{m+n}^{+},
$$

where we identify $P_{m+n}^{+}$with $\mathbb{Z}_{+}^{m} \times \mathbb{Z}_{+}^{n}$. Now, we can state a Weyl type formula for $\operatorname{ch} L_{m+n}(\Lambda(\lambda))$.

Theorem 4.10 (cf. $[12,14]$ ) Given $\Lambda \in P_{m+n}^{+}$with $\Lambda=\Lambda(\lambda)$ for some $\lambda \in \mathbb{Z}_{+}^{d}$, we have

$$
\operatorname{ch} L_{m+n}(\Lambda)=\sum_{w \in \mathcal{W}_{m+n}(\Lambda)}(-1)^{\ell(w)} \operatorname{ch} V_{m+n}(w * \Lambda)
$$

Proof It follows from Proposition 4.7 with Theorem 3.10. The proof is similar to that of Theorem 4.3.

Remark 4.11 The parametrization of highest weights for generalized Verma modules in Theorem 4.10 is different from the one in $[12,14]$, where the sum is given over 
the Weyl group of $\mathfrak{g l}_{m+n}$ with its shifted action on the highest weight $\Lambda$, which is not equal to $w * \Lambda$ for $w \in \mathcal{W}_{m+n}(\Lambda)$ by definition. It would be interesting to compare these two formulas.

Example 4.12 Suppose that $\lambda=\mathbf{0}_{d}$. Then $\Lambda\left(\mathbf{0}_{d}\right)=\left(-\left(d^{m}\right), \mathbf{0}_{n}\right)$ and each $w \in$ $\mathcal{W}_{m+n}\left(-\left(d^{m}\right), \mathbf{0}_{n}\right)$ of minimal length corresponds to a unique partition $\mu \subset\left(m^{n}\right)$ with $\ell(w)=|\mu|$ (see Remark $3.3(1))$. Hence, we have $w *\left(-\left(d^{m}\right), \mathbf{0}_{n}\right)=\left(-\left(d^{m}\right)-\right.$ $\left.\left(d^{\delta(\mu)}\right)-\mu^{\prime *}, \mu+\left(d^{\delta(\mu)}\right)\right)$, and

$$
\operatorname{ch} L_{m+n}\left(\Lambda\left(\mathbf{0}_{d}\right)\right)=\sum_{\mu \subset\left(m^{n}\right)}(-1)^{|\mu|} \frac{s_{\mu^{\prime}+\left(d^{m}\right)+\left(d^{\delta(\mu)}\right)}\left(\mathbf{x}_{[-m]}^{-1}\right) s_{\mu+\left(d^{\delta(\mu)}\right)}\left(\mathbf{x}_{[n]}\right)}{\prod_{i, j}\left(1-x_{i}^{-1} x_{j}\right)},
$$

where $i \in[-m]$ and $j \in[n]$.

Remark 4.13 We may apply Theorem 3.10 to other irreducible highest weight representations of a Lie (super)algebra $\mathfrak{g}$, whenever we have a Howe duality of $\left(\mathfrak{g}, \mathfrak{g l}_{d}\right)$. In fact, the associated irreducible characters for $\mathfrak{g}$ satisfy a Cauchy type identity of the form given in Theorem 3.10 and hence they are equal to $S_{\lambda}^{\mathcal{A} / \mathcal{B}}$ under suitable choices of $\mathcal{A}$ and $\mathcal{B}$ (see [27] for more examples).

Acknowledgements Part of this work was done during the author's visit at National Taiwan University in 2006 summer. He would like to thank Shun-Jen Cheng for the invitation and kind explanations on his recent works. He also thanks the referee for helpful suggestions on the first version of this manuscript.

\section{References}

1. Beilinson, A., Bernstein, J.: Localisation de g-modules. C. R. Acad. Sci. Paris Ser. I Math. 292, 15-18 (1981)

2. Brylinski, J.L., Kashiwara, M.: Kazhdan-Lusztig conjecture and holonomic systems. Invent. Math. 64, 387-410 (1981)

3. Berele, A., Regev, A.: Hook Young diagrams with applications to combinatorics and to the representations of Lie superalgebras. Adv. Math. 64, 118-175 (1987)

4. Björner, A., Brenti, F.: Combinatorics of Coxeter Groups. Graduate Texts in Mathematics, vol. 231. Springer, Berlin (2005)

5. Brundan, J.: Kazhdan-Lusztig polynomials and character formulae for the Lie superalgebra $\mathfrak{g l}(m \mid n)$. J. Am. Math. Soc. 16, 185-231 (2003)

6. Casian, L., Collingwood, D.: The Kazhdan-Lusztig conjecture for generalized Verma modules. Math. Z. 195, 581-600 (1987)

7. Cheng, S.-J., Wang, W.: Howe duality for Lie superalgberas. Compos. Math. 128, 55-94 (2001)

8. Cheng, S.-J., Wang, W.: Lie subalgebras of differential operators on the super circle. Publ. Res. Inst. Math. Sci. 39, 545-600 (2003)

9. Cheng, S.-J., Wang, W., Zhang, R.B.: A super duality and Kazhdan-Lusztig polynomials. arXiv:math.RT/0409016

10. Cheng, S.-J., Zhang, R.B.: Analogue of Kostant's u-cohomology formula for the general linear superalgebra. Int. Math. Res. Not. 31-53 (2004)

11. Cheng, S.-J., Zhang, R.B.: Howe duality and combinatorial character formula for orthosymplectic Lie superalgebras. Adv. Math. 182, 124-172 (2004)

12. Davidson, M., Enright, T., Stanke, R.: Differential operators and highest weight representations. Mem. Am. Math. Soc. 94, 455 (1991)

13. Deodhar, V.: On some geometric aspects of Bruhat orderings, II: the parabolic analogue of KazhdanLusztig polynomials. J. Algebra 111, 483-506 (1987) 
14. Enright, T.: Analogues of Kostant's u-cohomology formulas for unitary highest weight modules. J. Reine Angew. Math. 392, 27-36 (1988)

15. Enright, T., Howe, R., Wallach, N.: A classification of unitary highest weight modules, in Representation theory of reductive groups. Prog. Math. 40, 97-143 (1983)

16. Fulton, W.: Young Tableaux. London Mathematical Society Student Texts, vol. 35. Cambridge University Press, Cambridge (1997)

17. Frenkel, I.: Representations of Kac-Moody algebras and dual resonance models in Applications of group theory in physics and mathematical physics. In: Lectures in Applied Mathematics, vol. 21, pp. 325-353. AMS, Providence (1985)

18. Garland, H., Lepowsky, J.: Lie Algebra Homology and the Macdonald-Kac Formulas. Invent. Math. 34, 37-76 (1976)

19. Howe, R.: Remarks on classical invariant theory. Trans. Am. Math. Soc. 313, 539-570 (1989)

20. Kac, V.G.: Lie superalgebras. Adv. Math. 26, 8-96 (1977)

21. Kac, V.G.: Characters of typical representations of classical Lie superalgebras. Commun. Algebra $\mathbf{5}$, 889-897 (1977)

22. Kac, V.G.: Representations of classical Lie superalgebras in differential geometrical methods in mathematical physics II. In: Lecture Notes in Mathematics, vol. 676, pp. 597-626. Springer, Berlin (1978)

23. Kac, V.G., Radul, A.: Representation theory of the vertex algebra $W_{1+\infty}$. Transform. Groups 1, 41-70 (1996)

24. Kashiwara, M., Vergne, M.: On the Segal-Shale-Weil representations and harmonic polynomials. Invent. Math. 44, 1-47 (1978)

25. Kazhdan, D., Lusztig, G.: Representations of Coxeter groups and Hecke algebras. Invent. Math. 53, 165-184 (1979)

26. Knuth, D.: Permutations, matrices, and the generalized Young tableaux. Pac. J. Math. 34, 709-727 (1970)

27. Kwon, J.-H.: Rational semistandard tableaux and character formula for the Lie superalgebra $\widehat{\mathfrak{g l}}_{\infty \mid \infty}$. Adv. Math. 217, 713-739 (2008)

28. Kwon, J.-H.: Crystal graphs for Lie superalgebras and Cauchy decomposition. J. Algebr. Comb. 25, 57-100 (2007)

29. Macdonald, I.G.: Symmetric Functions and Hall Polynomials, 2nd edn. Oxford University Press, Oxford (1995)

30. Serganova, V.: Kazhdan-Lusztig polynomials and character formula for the Lie superalgebra $\mathfrak{g l}(m \mid n)$. Sel. Math. (N.S.) 2, 607-651 (1996)

31. Sergeev, A.N.: Tensor algebra of the identity representation as a module over the Lie superalgebras $\mathrm{Gl}(n, m)$ and $Q(n)$. Mat. Sb. (N.S.) 123(165), 422-430 (1984) 